\section{Una modificación del coeficiente alfa de Cronbach por errores correlacionados}

\section{A modification of Cronbach's alpha coefficient by correlated errors}

\section{Sr. Editor:}

La presente carta se centra en el impacto de los errores correlacionados en la estimación de la confiabilidad de los puntajes del Índice de Bienestar Personal (PWI-7), reportada en Oyanedel et $\mathrm{al}^{1}$. En este estudio se halló una confiabilidad satisfactoria del puntaje $(\alpha=0,85)$, pero en vista de la presencia de estos errores correlacionados, la confiabilidad está sobrestimada, tal como lo demostraremos más adelante. Los errores correlacionados están reportados en la figura $1(\mathrm{p} .1148)$ y son de magnitudes moderadas $(0,47,0,50,0,53, \mathrm{y} 0,56)$.

El coeficiente $\alpha^{2}$ es el estimador de la confiabilidad de los puntajes que se usa con mayor frecuencia, pero muchas veces se emplea sin considerar sus dos supuestos básicos: tau-equivalencia de los ítems y ausencia de errores correlacionados. Aunque generalmente estos requisitos son difíciles de lograr en la práctica ${ }^{3}$, la violación de cada uno de ellos sesga la estimación de este coeficiente. En cuanto al primero, se refiere a la similaridad de las medias (o cargas factorales) de los ítems; y el incumpliento de esta condición trae consigo la subestimación del $\alpha$, es decir, que se obtendrán coeficientes bajos. Con relación a la presencia de errores correlacionados, ésta produce una sobrestimación del coeficiente ${ }^{3-5}$, pues representa la varianza no relacionada con el constructo que se añade a la estimación de la confiabilidad. La violación simultánea de ambos supuestos da como resultado que el $\alpha$ sea considerado como el límite superior de la confiabilidad (upperbound) ${ }^{6}$. De acuerdo a la teoría de la medición, uno de sus presupuestos es que los errores (o varianza no relacionada con el constructo) entre los ítems son independientes entre $s^{3,3}$.

El incumplimiento del supuesto de errores independientes ha sido revisado anteriormente en la literatura especializada, concluyéndose que puede provenir de causas que van más allá del constructo que originalmente se pretende evaluar. Esto incluye, por ejemplo, factores de irrelevantes de contenido ${ }^{7}$, fraseo de los ítems ${ }^{5}$, cercanía de ítems en el protocolo de evaluación ${ }^{6}$, entre otros. Para controlar este impacto, se han desarrollado fórmulas de corrección del coeficiente $\alpha$ cuando sus ítems muestran errores correlacionados ${ }^{8-10}$. La más reciente ${ }^{9}$ proviene de la equivalencia entre el coeficiente $\alpha$ y el coeficiente Spearman-Brown (ecuación 1), y que es el fundamento para derivar una expresión matemática que considere los errores correlacionados (ecuación 2): 


$$
\begin{aligned}
& r_{X X}=\frac{k r_{i j}}{1+(k-1) r_{i j}} \\
& \alpha^{\prime}=\frac{k r_{i j}}{1+(k-1) \sqrt{r_{i j}^{2}+2 r_{e_{i j}^{2}}^{2}}}
\end{aligned}
$$

donde $k$ es el número de ítems, $r_{i j}$ es la correlación inter-ítem promedio, y $r_{e i j}$ es el promedio de la correlación entre errores. En base a dicha aproximación, se hizo un re-análisis considerando los datos de Oyanedel et al. ${ }^{1}$ para obtener una estimación menos sesgada del coeficiente $\alpha$. Si bien no se conoce $r_{\mathrm{ij}}$, se puede obtenerla usando la ecuación 1; teniendo en cuenta que $r_{x x}(0,85)$ y $k(7)$, el resultado es $r_{i j}=0,4474$. Por su parte, el promedio de errores correlacionados puede calcularse fácilmente $\left(r_{e i j}=0,515\right)$. Finalmente, aplicando la ecuación 2, el $\alpha$ 'es de 0,511; el intervalo de confianza ${ }^{11}$ de esta nueva estimación es $0,435-0,580$ ( $95 \%$ de confianza). La disminución obtenida es de $39,8 \%$, una cantidad que no podría considerarse insustancial por dos motivos: primero, incrementa el error aleatorio alrededor del puntaje individual de los sujetos evaluados por el instrumento. Y en segundo lugar, el estatus del coeficiente cambia desde un nivel que puede describirse como bueno, hasta un nivel que usualmente se describe como pobre ${ }^{12}$.

Efectivamente, la nueva magnitud obtenida es inaceptable incluso para propósitos de investigación y menos aún para propósitos clínicos, en que se requiere alta precisión en la toma de decisiones para el diagnóstico y determinación del cambio luego de una intervención terapéutica ${ }^{11,12}$. Generalmente, un valor de 0,90 es el mínimo requerido para tomar decisiones clínicas en sujetos, y algo menos para decisiones de grupo $^{6,11,12}$. Como se puede deducir, esto pone en tela de juicio las conclusiones presentadas por los autores, quienes afirmaron que la confiabilidad es adecuada. Más aún, el grado de confiabilidad corregida indica que el grado de error en el puntaje del PWI-7 afectará directamente a la validez del instrumento (menor a 0,70 ) ya todo análisis con base en esos datos (comparación de grupos, correlaciones, etc. $)^{6}$.

Los hallazgos en este re-análisis llevarían a los autores del estudio a una re-interpretación de la confiabilidad de los puntajes del PWI-7, así como de su dimensionalidad, dado que debe brindarse una explicación para la presencia de errores correlacionados que sea compatible con lo expresado en la literatura psicométrica. Por otro lado, es plausible que deba considerarse algún otro coeficiente alternativo como estimador de la confiabilidad del PWI-7, pues las diferencias en las magnitudes de las cargas factoriales de sus pueden re- presentar que la tau-equivalencia no se cumple. Otros coeficientes de confiabilidad, como el coeficiente omega o Feldt-Brennan ${ }^{6}$, pueden ser adecuados en lugar del coeficiente $\alpha$, pues no requieren del cumplimiento de esta característica.

\section{Sergio Alexis Dominguez-Lara ${ }^{1}$, César Merino-Soto ${ }^{1}$ ${ }^{1}$ Instituto de Investigación de Psicología, Universidad de San Martín de Porres}

\section{Referencias}

1. Oyanedel JC, Vargas S, Mella C, Páez D. Validación del índice de bienestar personal (PWI - 7) en usuarios vulnerables de servicios de salud en Santiago, Chile. Rev Med Chile 2015; 143: 1144-51.

2. Cronbach LJ. Coefficient alpha and the internal structure of tests. Psychometrika 1951; 16; 297-334.

3. Dunn TJ, Baguley T, Brunsden V. From alpha to omega: A practical solution to the pervasive problema of internal consistency estimation. Br J Psychol 2014; 105 (3): 399-412.

4. Green SB, Yang Y. Commentary on coefficient alpha: a cautionary tale. Psychometrika 2009; 74 (1): 121-35.

5. Yang Y, Green SB. A note on Structural Equation Modeling estimates of reliability. Struct Equat Modeling 2010; 17 (1): 66-81.

6. Meyer JP. Reliability. New York: Oxford University Press; 2010.

7. Raykov T. Coefficient alpha and composite reliability with interrelated non homogeneous items. App Psych Meas 1998; 22 (4): 375-85.

8. Komaroff E. Effect of simultaneous violations of esencial tau equivalence and uncorrelated error son coefficient alpha. App Psych Meas 1997; 21 (4): 337-48.

9. Pascual-Ferrá P, Beatty MJ. Correcting internal consistency estimates inflated by correlated item errors. Commun Res Rep 2015; 32 (4): 347-52.

10. Rae G. Correcting coefficient alpha for correlated errors: Is $\alpha \mathrm{k}$ a lower bound of reliability? App Psych Meas. 2006; 30 (1): 56-9.

11. Dominguez S, Merino C. ¿Por qué es importante reportar los intervalos de confianza del coeficiente alfa de Cronbach? Rev Latino Cien Social Niñez Juventud 2015; 13 (2): 1326-8.

12. Merino C, Navarro J, García W. Revisión de la consistencia interna del Inventario de Inteligencia Emcional de Bar-On, EQ-I: YV. Rev Per Psicol Trab Soc 2014; 3 (1): 141-54.

Correspondencia:

Sergio Dominguez Lara

Instituto de Investigación de Psicología, Universidad de San Martín de Porres.

Av. Tomás Marsano 242 (5to piso), Lima 34-Perú

sdominguezmpcs@gmail.com 